\title{
Field Corn Production Problems: A Diagnostic Guide ${ }^{1}$
}

\section{David Wright and Jim Rich ${ }^{2}$}

The field is a complex environment with many factors that can interact to influence the growth of a corn plant. These factors can be in the form of insects, diseases, nematodes and weeds (biotic); or, they can be factors such as weather, nutrients, or chemicals (abiotic). Under optimum conditions, production of field corn can exceed over 200 bushels of corn per acre; under totally unfavorable conditions, every corn plant can die.

This guide was prepared to help identify problems so the proper corrective measures can be taken to minimize or prevent yield losses. For any corrective action to be successful, early detection is essential.

The Florida Extension Plant Diagnostic Clinics can help determine disease and insect diagnostic problems that cannot be easily identified. The clinic works primarily through county extension offices in Florida. You may contact your county office with your unidentified disease or pest problem.

There are also web sites where pictures can be found such as <http://www.ent.iastate.edu/imagegal/plantpath/> or <http://www.ent.iastate.edu/imagegal/plantpath/corn/ ndeficiency/> and <http://www.ent.iastate.edu/imagegal/plantpath/corn/ kdeficiency/sawyer1mc-007.html>. Some problems (pest or nutrient) have similar symptoms and therefore observation alone as described in this document is not a definitive diagnosis, but serves as an initial guide.

\section{Growth Stage Definitions}

Before Emergence- Seed planted -Germination of seed may require from 4 to 30 days depending upon soil temperature.

Two Leaf Stage- Two leaves fully open, collar visible- Leaves have emerged but the growing point is still below the soil surface.

Early Whorl- 4-6 leaves fully emerged- Plants are in the 5 leaf stage and larger.

Mid Whorl- 8-10 leaves fully emerged -This is a period of rapid leaf formation and the beginning of rapid stalk elongation. The tassel and ear shoots are developing.

1. This document is SS-AGR-200, a publication of the Department of Agronomy, Florida Cooperative Extension Service, Institute of Food and Agricultural Sciences, University of Florida. Publication Date: February 2004. Please visit the EDIS website at http://edis.ifas.ufl.edu.

2. David Wright, professor, Department of Agronomy, North Florida Research and Education Center, Quincy, FL; Jim Rich, professor, Department of Entomology and Nematology, North Florida Research and Education Center, Quincy, FL; Florida Cooperative Extension Service, Institute of Food and Agricultural Sciences, University of Florida, Gainesville FL.

The use of trade names in this publication is solely for the purpose of providing specific information. UF/IFAS does not guarantee or warranty the products named, and references to them in this publication does not signify our approval to the exclusion of other products of suitable composition. Use herbicides safely. Read and follow directions on the manufacturer's label. 
Late Whorl- 12-16 leaves fully emerged- Leaf

enlargement is complete, and brace roots are developing. The potential numbers of kernels on the ear is determined by this time. The stalk is rapidly growing and the tassel is almost full size.

\section{Tasseling- Tassel emerging- Final stalk} elongation occurs during this stage.

\section{Early Silk- Silks emerging, pollen shedding-} The tassel is fully emerged, and stalk development is complete. The ear shank and husks are growing rapidly. Ovules are enlarging and the silk from each ovule is near the tip of the ear and emerging. The number of ovules that will be fertilized and develop into kernels is being determined at this stage.

Blister Stage- Brown silk, cob full size, watery kernels-The cob, husk and shank are fully developed. Starch has begun to accumulate and the kernels are rapidly increasing in size. The plants continue to absorb soil nitrogen and phosphorus, but much of these nutrients are being supplied from other plant parts. High water uptake period for grain fill.

\section{Soft Dough- Kernels milky with some starch-} Starch is accumulating and embryo formation in each new kernel is underway.

Hard Dough- Few kernels with dents- Embryo growth is rapid, and kernels are denting.

\section{Physiological Maturity- Black layer formed,} grain mature and drying- Dry matter accumulation has ceased, and moisture loss begins. The husks and some leaves are usually no longer green. Most kernels are dented, and "black layer" formation is complete. Moisture is between 30-35\%. 
Table 1. Diagnosis of General Plant Problems by Growth Phase.

\begin{tabular}{|c|c|c|}
\hline General Appearance & Specific Symptoms & Possible Cause(s) \\
\hline \multicolumn{3}{|l|}{ I. BEFORE EMERGENCE } \\
\hline \multirow[t]{2}{*}{$\begin{array}{l}\text { A. Skip in rows where plants fail to } \\
\text { emerge. }\end{array}$} & \multirow[t]{2}{*}{ Seed not sprouted. } & $\begin{array}{l}\text { Seed not viable Anhydrous or aqua } \\
\text { ammonia injury. }\end{array}$ \\
\hline & & $\begin{array}{l}\text { Excessive fertilizer (nitrogen and/or } \\
\text { potash) placed too close to seed. Soil } \\
\text { too dry, or water logged. }\end{array}$ \\
\hline \multirow[t]{4}{*}{ B. Seed or sprout eaten or dug up. } & Seed swollen, but not sprouted. & \multirow[t]{4}{*}{ Mice, skunk, rats, crows, blackbirds. } \\
\hline & Rotted seed or seedlings. & \\
\hline & Seed hollowed out. & \\
\hline & $\begin{array}{l}\text { Unemerged seedling dug up and/or } \\
\text { entire plant eaten. }\end{array}$ & \\
\hline \multicolumn{3}{|l|}{ II. EMERGENCE TO KNEE-HIGH } \\
\hline \multirow{4}{*}{$\begin{array}{l}\text { A. Scattered problem spots of dead } \\
\text { or poorly growing plants }\end{array}$} & Plants stunted, wilted and/or discolored. & \multirow[t]{4}{*}{ Cutworm damage. } \\
\hline & Plants cut off above or below ground. & \\
\hline & Sudden death of leaves or plants. & \\
\hline & $\begin{array}{l}\text { Plants with rough gouged out feeding } \\
\text { damage at or just below the soil } \\
\text { surface. }\end{array}$ & \\
\hline \multirow[t]{4}{*}{ B. Wilting } & $\begin{array}{l}\text { Upper leaves roll and appear dull or } \\
\text { sometimes purple; stunting of plants; } \\
\text { plants may die. }\end{array}$ & \multirow[t]{3}{*}{$\begin{array}{l}\text { Dry surface soil, shallow planting (1/2 } \\
\text { inch or less), sand blasting. }\end{array}$} \\
\hline & Whorl dead. & \\
\hline & Crown roots not developing. & \\
\hline & $\begin{array}{l}\text { Plants appear weak and sickly; wilting } \\
\text { may occur; tunneling or girdling at or } \\
\text { near soil surface is apparent. }\end{array}$ & $\begin{array}{l}\text { Lesser corn stalk borer caused by dry } \\
\text { weather or planting into not fully } \\
\text { decayed or green plant material. }\end{array}$ \\
\hline \multirow[t]{8}{*}{ C. Plants discolored. } & $\begin{array}{l}\text { Leaves appear sandblasted; leaves } \\
\text { pale-green or whitish in color. }\end{array}$ & \multirow[t]{2}{*}{$\begin{array}{l}\text { Potassium deficiency. Excessive } \\
\text { fertilizer or chemical uptake. }\end{array}$} \\
\hline & Leaf edges yellow or dead. & \\
\hline & $\begin{array}{l}\text { Yellowing or browning beginning from } \\
\text { the tips. }\end{array}$ & $\begin{array}{l}\text { Excessive herbicide rates-(linuron } \\
\text { (Lorox), ametryn (Evik), post } \\
\text { emergence with oil, atrazine and other } \\
\text { photosynthetic inhibitors: in severe } \\
\text { cases, plants may die). }\end{array}$ \\
\hline & $\begin{array}{l}\text { Lower leaves dead, tips on upper } \\
\text { leaves dying. }\end{array}$ & Fertilizer injury. \\
\hline & General yellowing of upper leaves. & Sulfur deficiency. \\
\hline & General yellowing of lower leaves. & $\begin{array}{l}\text { Excessive moisture. Nitrogen } \\
\text { deficiency. }\end{array}$ \\
\hline & $\begin{array}{l}\text { Purpling or reddening of leaves from } \\
\text { tips backwards; affects lower leaves } \\
\text { initially; leaf tips may later turn dark } \\
\text { brown and die. }\end{array}$ & Phosphorus deficiency. \\
\hline & Leaves slowly turn white to tan and die. & $\begin{array}{l}\text { Glyphosate (Roundup), other } \\
\text { herbicide injury. }\end{array}$ \\
\hline
\end{tabular}


Table 1. Diagnosis of General Plant Problems by Growth Phase.

\begin{tabular}{|c|c|c|}
\hline General Appearance & Specific Symptoms & Possible Cause(s) \\
\hline & $\begin{array}{l}\text { Irregular light and dark mottling or } \\
\text { mosaic on the leaves. Sometimes } \\
\text { reddening of leaves. }\end{array}$ & \multirow[t]{2}{*}{$\begin{array}{l}\text { Maize dwarf mosaic or maize chlorotic } \\
\text { dwarf viruses. }\end{array}$} \\
\hline & $\begin{array}{l}\text { Irregular light gray or silvery blotches } \\
\text { on both sides of leaves on the east side } \\
\text { of affected plants. }\end{array}$ & \\
\hline & $\begin{array}{l}\text { Light streaking of leaves which } \\
\text { develops into a broad band of bleached } \\
\text { tissue on each side of the midribs; } \\
\text { leaf-midribs and margins remain green; } \\
\text { sometimes stalks and leaf edges } \\
\text { appear to be tinted red or brown. }\end{array}$ & Zinc deficiency. \\
\hline & $\begin{array}{l}\text { Bright yellow to white strips with } \\
\text { smooth margins running the length of } \\
\text { leaves; may appear on scattered plants } \\
\text { throughout the field and sometimes, } \\
\text { only on one side of a plant. }\end{array}$ & Genetic stripes. \\
\hline & $\begin{array}{l}\text { White or yellow stripes between leaf } \\
\text { veins. }\end{array}$ & $\begin{array}{l}\text { Excessively acidic soil; Magnesium, } \\
\text { iron, manganese, boron or sulfur } \\
\text { deficiencies. }\end{array}$ \\
\hline & $\begin{array}{l}\text { Distinct bleached bands across leaf } \\
\text { blades; leaf tissue may collapse at } \\
\text { discolored bands, resulting in the leaf } \\
\text { folding downward at this point. }\end{array}$ & $\begin{array}{l}\text { Air pollution injury; herbicides injury; } \\
\text { cold banding. }\end{array}$ \\
\hline \multirow[t]{2}{*}{ D. Plants discolored and stunted. } & \multirow[t]{2}{*}{$\begin{array}{l}\text { Leaves yellow; plants spindly and } \\
\text { stunted. }\end{array}$} & $\begin{array}{l}\text { Nitrogen deficiency. Sulfur } \\
\text { deficiency-more pronounced on } \\
\text { younger leaves than nitrogen } \\
\text { deficiency; Water logged soils, sting or } \\
\text { stubby-root nematodes. }\end{array}$ \\
\hline & & Phosphorus deficiency-mild. \\
\hline
\end{tabular}


Table 1. Diagnosis of General Plant Problems by Growth Phase.

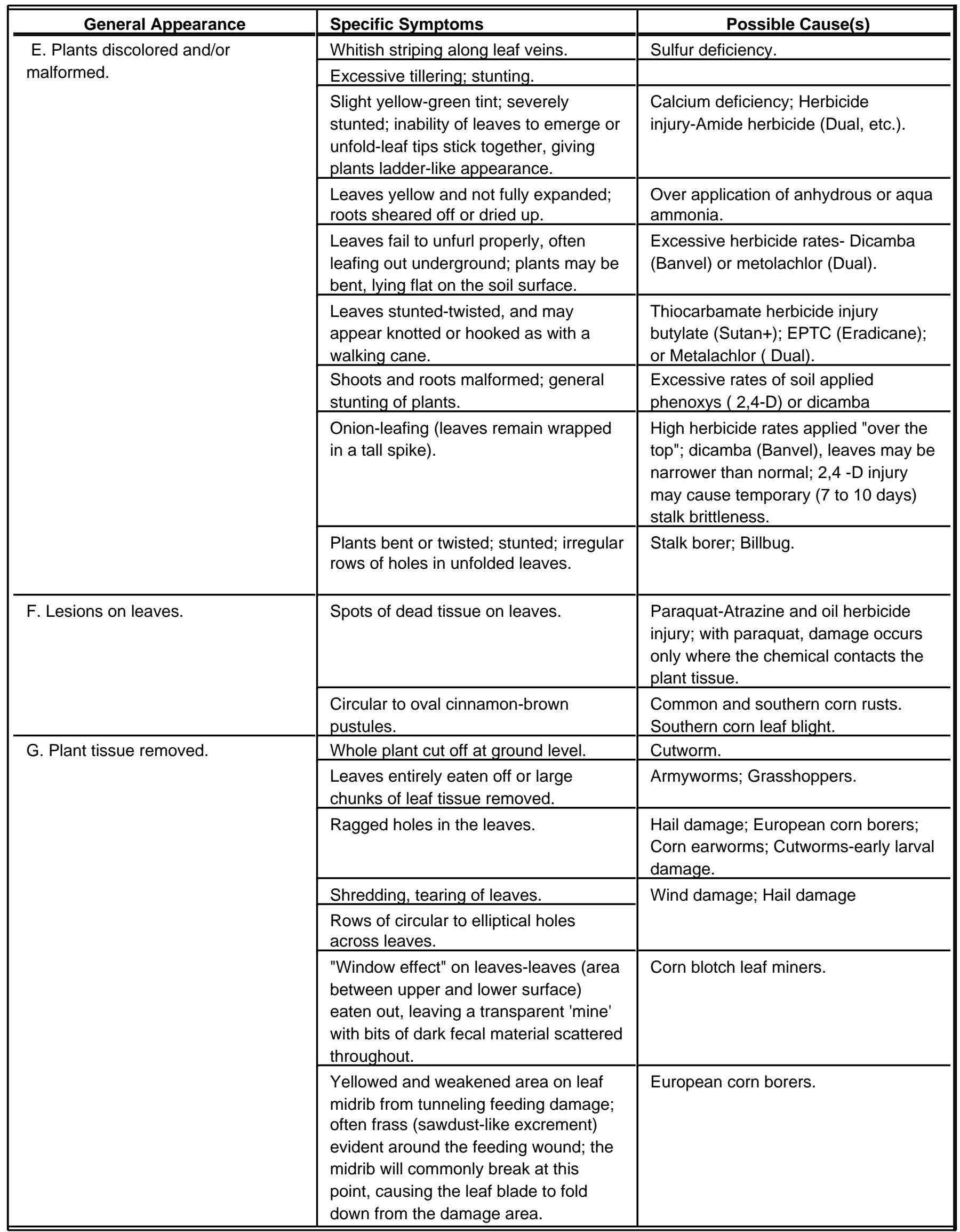


Table 1. Diagnosis of General Plant Problems by Growth Phase.

\begin{tabular}{|c|c|c|}
\hline General Appearance & Specific Symptoms & Possible Cause(s) \\
\hline \multicolumn{3}{|l|}{ III. KNEE-HIGH TO TASSELING } \\
\hline \multirow[t]{3}{*}{$\begin{array}{l}\text { A. Severe wilting and/or death of } \\
\text { plants. }\end{array}$} & Sudden death of plants. & $\begin{array}{l}\text { Lightning (All plant material in an } \\
\text { approximately circular area suddenly } \\
\text { killed; plants along margin of affected } \\
\text { area may be severely to slightly } \\
\text { injured; severely injured plants may die } \\
\text { later). }\end{array}$ \\
\hline & $\begin{array}{l}\text { Dieback of leaves wilting, then drying } \\
\text { up of leaf tissue, beginning at leaf tips. }\end{array}$ & $\begin{array}{l}\text { Molybdenum deficiency (younger } \\
\text { leaves may twist); Air pollution }\end{array}$ \\
\hline & $\begin{array}{l}\text { Dead leaves or plants, usually at row } \\
\text { ends. }\end{array}$ & $\begin{array}{l}\text { Excessive fertilizer or herbicide rates, } \\
\text { or soil compaction. }\end{array}$ \\
\hline \multirow[t]{9}{*}{ B. Plants discolored } & $\begin{array}{l}\text { Yellowing of plants, beginning with } \\
\text { lower leaves. }\end{array}$ & \multirow[t]{2}{*}{ Potassium deficiency. } \\
\hline & $\begin{array}{l}\text { Yellowing of leaf margins beginning at } \\
\text { tips; affected tissue later turns brown } \\
\text { and dies. }\end{array}$ & \\
\hline & $\begin{array}{l}\text { Purpling or reddening of leaves from tip } \\
\text { backward; affects lower leaves initially; } \\
\text { leaf tips may turn dark brown and die. }\end{array}$ & Phosphorus deficiency. \\
\hline & \multirow[t]{2}{*}{$\begin{array}{l}\text { Yellow to white interveinal striping on } \\
\text { leaves. }\end{array}$} & $\begin{array}{l}\text { Genetic stripe-(stripes have smooth } \\
\text { margins; may appear on scattered } \\
\text { plants throughout the field and, } \\
\text { sometimes, only one side of a plant). }\end{array}$ \\
\hline & & $\begin{array}{l}\text { Boron deficiency-(initially white, } \\
\text { irregularly shaped spots develop } \\
\text { between veins which may coalesce to } \\
\text { form white stripes that appear waxy } \\
\text { and raised from leaf surface; plants } \\
\text { may be stunted). }\end{array}$ \\
\hline & Leaves completely white. & Herbicides such as Zorial or Cotoran. \\
\hline & Leaves turn whitish. & Spider mites. \\
\hline & $\begin{array}{l}\text { Pale green to white stripes between } \\
\text { leaf veins, usually on upper leaves. }\end{array}$ & Iron deficiency. \\
\hline & $\begin{array}{l}\text { Upper leaves show pale green to yellow } \\
\text { interveinal discoloration; lower leaves } \\
\text { appear olive green and somewhat } \\
\text { streaked; severe damage appears as } \\
\text { elongated white streaks. The center of } \\
\text { which turns brown and falls out. }\end{array}$ & Manganese deficiency. \\
\hline \multirow[t]{2}{*}{ C. Plants discolored and malformed. } & $\begin{array}{l}\text { Plants show stunting and/or a mottle or } \\
\text { fine chlorotic strips in leaves. Leaves } \\
\text { can be reddish. }\end{array}$ & $\begin{array}{l}\text { Maize dwarf mosaic virus or maize } \\
\text { dwarf chlorotic virus. }\end{array}$ \\
\hline & $\begin{array}{l}\text { Stunting, tillering; twisting and rolling of } \\
\text { leaves. }\end{array}$ & Crazy top, plant virus. \\
\hline
\end{tabular}


Table 1. Diagnosis of General Plant Problems by Growth Phase.

\begin{tabular}{|c|c|c|}
\hline General Appearance & Specific Symptoms & Possible Cause(s) \\
\hline & $\begin{array}{l}\text { Plants darker green; stalks twisted at } \\
\text { mid to upper half; ears often do not } \\
\text { develop. }\end{array}$ & \multirow[t]{3}{*}{$\begin{array}{l}\text { Herbicide injury-butlylate (Sutan+) or } \\
\text { EPTC (Eradicane). }\end{array}$} \\
\hline & $\begin{array}{l}\text { Twisting and bending at corn stalks } \\
\text { above the ear shoot; ear may be } \\
\text { malformed. }\end{array}$ & \\
\hline & $\begin{array}{l}\text { Plants lodge or grow up in a curved } \\
\text { "sled runner" or gooseneck shape. }\end{array}$ & \\
\hline & $\begin{array}{l}\text { Soft, glistening white gall soon } \\
\text { becomes black and dusty on stalks, } \\
\text { leaves, ear or tassel. }\end{array}$ & Common smut (fungus). \\
\hline \multirow[t]{5}{*}{ E. Plant tissue removed. } & $\begin{array}{l}\text { Ragged holes in the leaves and } \\
\text { shredding of plants. }\end{array}$ & Hail damage. \\
\hline & Shredding, tearing of leaves. & Wind damage. \\
\hline & $\begin{array}{l}\text { Leaf tissue skeletonized or lacy in } \\
\text { appearance. }\end{array}$ & Japanese beetles. \\
\hline & $\begin{array}{l}\text { Leaves entirely eaten off or large } \\
\text { chunks of leaf tissue removed. }\end{array}$ & \multirow{2}{*}{$\begin{array}{l}\text { Armyworms. Grasshoppers. Fall } \\
\text { armyworms. Corn earworms. } \\
\text { Livestock. Wildlife. }\end{array}$} \\
\hline & $\begin{array}{l}\text { Holes bored into stalks and area within } \\
\text { stalk hollowed out by feeding } \\
\text { damage. }\end{array}$ & \\
\hline \multirow[t]{2}{*}{ F. Lesions on plants. } & $\begin{array}{l}\text { Oval, circular, or rectangular lesions, } \\
\text { tan to brown in color. }\end{array}$ & Southern corn leaf blight. \\
\hline & $\begin{array}{l}\text { Circular to oval, brick-red to } \\
\text { cinnamon-brown pustules on leaves. }\end{array}$ & $\begin{array}{l}\text { Common and southern corn rust } \\
\text { (fungi). }\end{array}$ \\
\hline \multicolumn{3}{|l|}{ IV. TASSELING TO MATURITY } \\
\hline \multirow[t]{2}{*}{ A. Silking impaired. } & Delayed silking or failure to silk. & \multirow{2}{*}{$\begin{array}{l}\text { Cucumber beetles. Japanese beetles. } \\
\text { Grasshoppers. }\end{array}$} \\
\hline & Silks clipped off. & \\
\hline \multirow[t]{4}{*}{ B. Tassels malformed. } & Tassels fail to emerge. & $\begin{array}{l}\text { Boron deficiency-(initially white, } \\
\text { irregularly-shaped spots develop } \\
\text { between veins which may coalesce } \\
\text { and form white stripes that appear } \\
\text { waxy and raised from leaf surface; } \\
\text { plants may be stunted). }\end{array}$ \\
\hline & $\begin{array}{l}\text { Tassels and upper stalk and foliage } \\
\text { bleached-premature drying. }\end{array}$ & Anthracnose. \\
\hline & Tassels develop as a mass of leaves. & Crazy top a plant virus. \\
\hline & Broken tassel from tunnelling. & European corn borer. \\
\hline \multirow[t]{3}{*}{ C. Plants discolored. } & $\begin{array}{l}\text { Yellowing of leaf margins beginning at } \\
\text { tips; affected tissue later turns brown } \\
\text { and dies. }\end{array}$ & Potassium deficiency. \\
\hline & Reddish or purple leaf. & $\begin{array}{l}\text { Injury near leaf base, leaf injury; no ear } \\
\text { on stalks and leaf is a carbohydrate } \\
\text { sink. }\end{array}$ \\
\hline & $\begin{array}{l}\text { Irregular, purple-brown spots or } \\
\text { blotches on sheaths. }\end{array}$ & Purple sheath spots. \\
\hline
\end{tabular}


Table 1. Diagnosis of General Plant Problems by Growth Phase.

\begin{tabular}{|c|c|c|}
\hline General Appearance & Specific Symptoms & Possible Cause(s) \\
\hline \multirow[t]{2}{*}{$\begin{array}{l}\text { D. Stalks malformed and/ or } \\
\text { broken. }\end{array}$} & $\begin{array}{l}\text { Lower stalk internodes and brace roots } \\
\text { easily compressed; stalks may lodge. }\end{array}$ & Fungal stalk rots. \\
\hline & Plants lodge, stalk may break. & $\begin{array}{l}\text { European corn borer; wind; potassium } \\
\text { deficiency-yellowing of leaf margins, } \\
\text { beginning at tips; affected tissue later } \\
\text { turns brown and dies. }\end{array}$ \\
\hline \multirow{3}{*}{$\begin{array}{l}\text { E. Premature death of all or some } \\
\text { parts of plants. }\end{array}$} & Sudden death of entire plant. & \multirow[t]{2}{*}{ Anthracnose leaf blight (fungus). } \\
\hline & $\begin{array}{l}\text { Extensive areas of leaf tissue die } \\
\text { prematurely, resulting in leaf drying. }\end{array}$ & \\
\hline & $\begin{array}{l}\text { Top kill-premature death of all or } \\
\text { portion of plants above ears. }\end{array}$ & Severe drought. \\
\hline \multirow{2}{*}{ F. Leaf tissue removed. } & Small, irregular holes in leaves. & European corn borer. \\
\hline & Large, irregular holes in leaves. & Grasshoppers; Fall armyworms. \\
\hline \multirow[t]{2}{*}{ G. Plants discolored or stunted. } & $\begin{array}{l}\text { Slight to severe stunting; yellowing and } \\
\text { sometimes reddening of foliage. }\end{array}$ & $\begin{array}{l}\text { Maize dwarf mosaic or Maize chlorotic } \\
\text { dwarf viruses. }\end{array}$ \\
\hline & Reddish or purple stalk & Barren plant. \\
\hline \multirow[t]{2}{*}{ H. Lesions on plants. } & $\begin{array}{l}\text { Tan, oval to rectangular lesions, tan to } \\
\text { brown in color. }\end{array}$ & Southern corn leaf blight. \\
\hline & $\begin{array}{l}\text { Elongate, irregular brown water-soaked } \\
\text { leaf stripes or spots on lower leaves. }\end{array}$ & Bacterial leaf spot. \\
\hline \multirow{5}{*}{ I. Damage to ears. } & $\begin{array}{l}\text { Tunneling or chewing feeding on } \\
\text { kernels. }\end{array}$ & $\begin{array}{l}\text { Corn earworm usually feed near the } \\
\text { ear tip; European corn borer-bore into } \\
\text { kernels and cobs; fall armyworms; } \\
\text { cucumber beetles; corn sap beetles. }\end{array}$ \\
\hline & Ears drop to ground. & $\begin{array}{l}\text { European corn borers-(feeding } \\
\text { damage to ear shanks and hybrid is } \\
\text { susceptible to ear drop). }\end{array}$ \\
\hline & Barren stalks. & $\begin{array}{l}\text { Maize dwarf mosaic or maize chlorotic } \\
\text { dwarf viruses; High temperatures } \\
\text { during pollination causing sterility; } \\
\text { Drought; Timing of silking not } \\
\text { synchronized with pollen shed; Plant } \\
\text { population too high; Low fertility; Silks } \\
\text { clipped back by insects; Corn leaf } \\
\text { aphids-caused delay or failure of } \\
\text { silking; } 2,4-D \text { herbicide injury-applied } \\
\text { during tasseling or pollen shed stage. }\end{array}$ \\
\hline & $\begin{array}{l}\text { Excessive ear shoots which are leafy } \\
\text { and barren. }\end{array}$ & Crazy top plant virus. \\
\hline & $\begin{array}{l}\text { Soft, glistening galls on the ear, later } \\
\text { black and powdery. }\end{array}$ & Common smut fungus. \\
\hline
\end{tabular}


Table 2. Herbicide Injury Guide.

\begin{tabular}{|c|c|c|}
\hline $\begin{array}{l}\text { Herbicide U.S. Common and } \\
\text { Trade Name }\end{array}$ & Symptoms & Remarks \\
\hline $\begin{array}{l}\text { Triazine (several products } \\
\text { simazine (Princep), ametryn } \\
\text { (Evik), etc. }\end{array}$ & $\begin{array}{l}\text { Gradual interveinal chlorosis. Leaves } \\
\text { may die back form tips and turn light } \\
\text { brown. Height of plants may be highly } \\
\text { variable. Where severe, entire plants } \\
\text { may be killed. Ametryn applied within } 3 \\
\text { weeks before tasseling may affect } \\
\text { pollination. }\end{array}$ & $\begin{array}{l}\text { Injury may occur on sandy soils low in } \\
\text { organic matter or due to excessive } \\
\text { rates. Cool, wet weather, or other } \\
\text { factors adversely affecting plant's } \\
\text { metabolism. }\end{array}$ \\
\hline $\begin{array}{l}\text { Acetanilides } \\
\text { metolachlor (Dual), propachlor } \\
\text { (Ramrod, ), etc. }\end{array}$ & $\begin{array}{l}\text { Injured seedlings and older plants may } \\
\text { have stunted or malformed shoots that } \\
\text { fail to unfurl. Plants may tend to leaf out } \\
\text { underground; may have some } \\
\text { "laddering". }\end{array}$ & $\begin{array}{l}\text { Cool, wet weather prior to emergence } \\
\text { is usually associated with injury, but } \\
\text { the majority of plants will grow out of } \\
\text { the damage. }\end{array}$ \\
\hline $\begin{array}{l}\text { Thiocarbamates products; } \\
\text { Butylate, (Sutan), EPTC } \\
\text { (Eptam, Eradicane), vernolate } \\
\text { (Vernam), etc. }\end{array}$ & $\begin{array}{l}\text { Extreme stunting, twisting, bending and } \\
\text { malformation of plants. Expanding } \\
\text { leaves may rupture and shred. Ear } \\
\text { malformation may occur. }\end{array}$ & $\begin{array}{l}\text { A "safening agent" added to } \\
\text { formulations reduces injury to maize. } \\
\text { Hybrids differ in sensitivity. }\end{array}$ \\
\hline 2,4-D products. & $\begin{array}{l}\text { Pre-emergence applications may } \\
\text { produce severe stunting and } \\
\text { malformation of roots and shoots. } \\
\text { Post-emergence directed sprays may } \\
\text { cause fasciation and upcurling of the } \\
\text { brace roots. 2,4-D applied } \\
\text { "over-the-top", especially on a hot day, } \\
\text { may cause stalks to be brittle and break. } \\
\text { Occasionally, a new leaf will fail to unfurl } \\
\text { (onion-leaf). Applied near tasseling or at } \\
\text { silking time, 2,4-D may interfere with } \\
\text { seed set. }\end{array}$ & $\begin{array}{l}\text { Do not apply } 2,4-D \text { with atrazine and } \\
\text { oil. Temperature and humidity at or } \\
\text { near time of application, growth stage } \\
\text { of maize, genetic susceptibility } \\
\text { method of application, and rate all } \\
\text { influence amount of post-emergence } \\
\text { injury. To avoid breakage, avoid } \\
\text { cultivating while plants are brittle. }\end{array}$ \\
\hline $\begin{array}{l}\text { Benzoic acid products- } \\
\text { Dicamba (Banvel), etc. }\end{array}$ & $\begin{array}{l}\text { Misapplication may cause onion leafing, } \\
\text { proliferation of inhibited roots, abnormal } \\
\text { brace root formation or fasciation. } \\
\text { Lodging may occur from } \\
\text { post-emergence applications. } \\
\text { Resembles 2,4-D injury symptoms. }\end{array}$ & $\begin{array}{l}\text { Dicamba applied pre-emergence } \\
\text { may cause injury, especially when } \\
\text { unfavorable environmental } \\
\text { conditions exist during seedling } \\
\text { emergence. }\end{array}$ \\
\hline $\begin{array}{l}\text { Phenylureas products- } \\
\text { Linuron (Lorox), etc }\end{array}$ & $\begin{array}{l}\text { Injury is similar to that caused by triazine } \\
\text { herbicides. Yellowing occurs first at leaf } \\
\text { tips and margins followed by browning. } \\
\text { Entire leaves may turn yellow and die. }\end{array}$ & $\begin{array}{l}\text { This compound is taken up by the } \\
\text { roots and translocated to the foliage. } \\
\text { Can also affect plant growth if } \\
\text { applied post-emergence. }\end{array}$ \\
\hline $\begin{array}{l}\text { Bipyridilium products- } \\
\text { (Paraquat, Gramoxone), etc. }\end{array}$ & $\begin{array}{l}\text { Leaf tissue turns brown and dies in } \\
\text { flecks or spots where contacted by spray } \\
\text { droplets; may superficially resemble } \\
\text { bacterial or fungal leaf blight. }\end{array}$ & $\begin{array}{l}\text { A non-selective contact herbicide } \\
\text { with no significant soil activity. Not } \\
\text { commonly recommended for directed } \\
\text { post-emergence use in corn. }\end{array}$ \\
\hline $\begin{array}{l}\text { Dinitroanalin products- } \\
\text { Pendimethalin (Prowl), trifluralin } \\
\text { (Treflan), etc. }\end{array}$ & $\begin{array}{l}\text { Reduced stand, stunting, or uneven } \\
\text { plant height, purpling of leaves with roots } \\
\text { somewhat pruned and "clubby" at ends. } \\
\text { Injury from pendimethalin may result } \\
\text { from direct pre-plant application. }\end{array}$ & $\begin{array}{l}\text { Incorporation of pendimethalin } \\
\text { increases risk of injury. May be } \\
\text { confused with injury from } \\
\text { nematodes. Major damage is from } \\
\text { carryover residue; chemicals differ in } \\
\text { their persistence. }\end{array}$ \\
\hline
\end{tabular}


Table 3. Guide to Nutrient Deficiency Symptoms of Corn.

\begin{tabular}{|c|c|}
\hline I. Stunted Plant & common to all deficiencies \\
\hline II. Loss of Green Color & common to all deficiencies \\
\hline A. Color changes in lower leaves: & Element Deficient \\
\hline $\begin{array}{l}\text { 1. Yellow discoloration from tip backward } \\
\text { in form of a } \mathrm{V}\end{array}$ & Nitrogen \\
\hline $\begin{array}{l}\text { 2.Brown discoloration and scorching along outer } \\
\text { margin from tip to base }\end{array}$ & Potassium \\
\hline $\begin{array}{l}\text { 3. Yellow discoloration between veins, finally edges } \\
\text { become reddish-purple }\end{array}$ & Magnesium \\
\hline $\begin{array}{l}\text { 4.Purpling and browning from tip backward, } \\
\text { waves }\end{array}$ & Phosphorus \\
\hline \multicolumn{2}{|l|}{ B. Color changes in upper leaves: } \\
\hline $\begin{array}{l}\text { 1. Emerging leaves show yellow to white bleached } \\
\text { bands in lower part of leaf }\end{array}$ & Zinc \\
\hline $\begin{array}{l}\text { 2. Young leaves show interveinal chlorosis } \\
\text { along entire length of leaf }\end{array}$ & Iron \\
\hline $\begin{array}{l}\text { 3. Young leaves uniformly pale yellow, older } \\
\text { leaves dying at the tips }\end{array}$ & Copper \\
\hline 4. White, irregular spots between veins & Boron \\
\hline $\begin{array}{l}\text { 5. Young leaves show pale green to yellow } \\
\text { discoloration between veins }\end{array}$ & Manganese \\
\hline 6. Young leaves wilt and die along the margins & Molybdenum \\
\hline $\begin{array}{l}\text { 7. Upper leaves usually paler than lower leaves } \\
\text { but can be uniform. Often develops stripping } \\
\text { between the veins }\end{array}$ & Sulfur \\
\hline
\end{tabular}

